http://dx.doi.org/10.21929/abavet2019.917

\title{
Uso de extractos de plantas en la fermentación ruminal in vitro
}

\author{
Use of plant extracts in vitro ruminal fermentation
}

\author{
Lucía Delgadillo-Ruiz1*@delgadillolucia@gmail.com, Rómulo Bañuelos-Valenzuela3** \\ (1) apozolero@hotmail.com, Francisco Echavarría-Cháirez²@ fechava1@yahoo.com, \\ Perla Ivonne Gallegos-Flores ${ }^{10}$ perla_gf17@hotmail.com, Carlos Meza-López ${ }^{3}$ \\ carmezlop@yahoo.com.mx
}

${ }^{1}$ Unidad Académica de Ciencias Biológicas, Universidad Autónoma de Zacatecas. Avenida preparatoria s/n colonia Hidráulica, CP. 98068, Zacatecas, Zacatecas, México. ${ }^{2}$ Instituto Nacional de Investigaciones Forestales, Agrícolas y Pecuarias. Campo experimental Zacatecas, México. ${ }^{3}$ Unidad Académica de Medicina Veterinaria y Zootecnia, Universidad Autónoma de Zacatecas. Carretera Panamericana FresnilloZacatecas s/n, Centro, CP. 98500 Víctor Rosales, Zacatecas, México. *Autor responsable: luciadelgadillo@uaz.edu.mx **Autor de correspondencia: apozolero@hotmail.com

\section{RESUMEN}

El objetivo fue obtener y caracterizar los extractos de Larrea tridentata, Origanum vulgare, Artemisa ludoviciana y Ruta graveolens para utilizarlos en la digestibilidad ruminal in vitro y cuantificar su producción de gas, ácidos grasos volátiles y de metano. Se determinó la composición química de los extractos por cromatografía de gases y para la producción de gas in vitro se utilizó fluido ruminal. La determinación de ácidos grasos volátiles se efectuó por cromatografía de gases. El metano se infirió con la concentración de ácidos grasos volátiles. Para la composición química se identificaron en los extractos los compuestos: terpineno, limoneno, linalool, timol y carvacrol. En la digestibilidad el que menor concentración de gas produjo a un volumen de $100 \mu \mathrm{L}$ de extracto fue $L$. tridentata y el metano se reporta 1.4 veces más que el control de alfalfa $\left(0.514 \mathrm{~mL} \mathrm{~g}^{-1}\right)$. Para la concentración de $330 \mu \mathrm{L}$ del extracto $O$. vulgaris, presentó inhibición de metano con respecto al control de alfalfa. Se concluye que el extracto cuatro de $O$. vulgaris fue el que presentó una mejor digestibilidad con respecto a la producción de gas, AGV (ácidos grasos volátiles) y menor concentración de metano con respecto al control.

Palabras clave: extractos de plantas, Ácidos grasos volátiles y Metano.

\section{ABSTRACT}

The objective was to obtain and characterize plant extracts of Larrea tridentata, Origanum vulgare, Artemisa ludoviciana and Ruta graveolens to be used in ruminal digestibility in vitro and to quantify the production of gas, volatile fatty acids and of methane. The chemical composition of the extracts was determinate by gas chromatography. Ruminal fluid was estimated according to Theodorou methodology for in vitro gas production. The determination of volatile fatty acids was carried out by gas chromatography. Methane was inferred from the concentration of volatile fatty acids. For the chemical composition, the following compounds was identified in the extracts: terpinene, limonene, linalool, thymol and carvacrol. In the digestibility that less gas produced at a volume of $100 \mu \mathrm{L}$ of extract was L. tridentata and methane is reported 1.4 times more than the standard of alfalfa $\left(0.514 \mathrm{~mL} \mathrm{~g}^{-1}\right)$. For the concentration of $330 \mu \mathrm{L}$ of the extract $\mathrm{O}$. vulgaris was present inhibition of methane with respect to the control of alfalfa. This concluded that the extract four of $O$. vulgaris was the one that presented a better digestibility with respect to gas production, AGV and lower concentration of methane with respect to the control.

Keywords: Plant extracts, Volatile fatty acids and Methane. 


\section{INTRODUCCIÓN}

El metano $\left(\mathrm{CH}_{4}\right)$, producido por los rumiantes domésticos representa una pérdida del 2 al $12 \%$ de la ingesta de energía bruta (EB) (Hristov et al., 2015, Moumen et al., 2016), ineficiencia importante en los sistemas de producción de estos (Moss et al., 2000, Moumen et al., 2016). Un mol de $\mathrm{CH}_{4}$, tiene la misma EB como para eliminar acetato; es decir $0.88 \mathrm{MJ} \mathrm{mol}^{-1}$, así la energía metabolizable neta de cualquier dieta aumenta el poder reductor de la formación $\mathrm{CH}_{4}$ en los productos finales que son utilizables por el rumiante. Un ovino adulto consume $22 \mathrm{Kg}$ materia seca (MS), con pérdida promedio de energía bruta (EB) del $6 \%$, y produce alrededor de $27 \mathrm{~mol} \mathrm{CH}_{4}$, que equivale a $2.5 \mathrm{~kg}$ de glucosa fermentada a $27 \mathrm{~mol}$ de acetato (Johnson et al., 1991, Moss et al., 2000).

La fermentación y degradación ruminal es principalmente el resultado de la actividad de bacterias, protozoos y hongos que habitan en el rumen del animal, los cuales actúan sobre las diferentes fracciones que componen el alimento. De manera particular, esta microflora ruminal hace posible que la fracción fibrosa ingerida que no puede ser enzimáticamente digerida por el animal, sea degradada y fermentada hacia ácidos grasos volátiles, gases de fermentación y calor; o incorporada a la materia microbiana digestible (Alejos et al., 2018).

Galicia et al. (2017), señalan que la capacidad de adaptarse rápidamente a los cambios del entorno es una de las principales características de la célula bacteriana; el rumen es un entorno altamente dinámico, y ninguno de los cambios es permanente, debido a las diversas especies microbianas que se encuentran en el rumen. Redes de transducción de señales, son las vías de procesamiento de información que reconocen diversos estímulos físicos y químicos, amplificación, procesamiento de señales y que desencadenan las respuestas de adaptación de la célula bacterianas.

Getachew et al. (2005) y Tapio (2017), mencionan que la fermentación microbiana que ocurre en el rumen, genera productos que pueden ser aprovechables por el animal; tales como los ácidos grasos volátiles, proteína microbiana y otro tipo de productos que se consideran desechos, como el dióxido de carbono y el metano. La fermentación microbiana de la fibra comprende varias acciones secuenciales, las cuales son señaladas por France (2005) y éstas son: hidratación, adherencia de los microorganismos apropiados, liberación de una mezcla de enzimas hidrolíticas y finalmente hidrólisis. La liberación resultante de monómeros es seguida por su posterior degradación intracelular en ácidos grasos volátiles (AGV) y gases de fermentación.

Debe considerarse que, junto a los carbohidratos, la fermentación de los lípidos y proteína de la ración también contribuyen en la producción de ácidos grasos volátiles; siendo menor para los lípidos y considerable para las proteínas cuando las dietas tienen alto contenido de proteína degradable en el rumen (Popova et al., 2017). Para la población 
microbiana, la mayoría de estos AGV constituyen productos de desecho; pero para el rumiante representan la principal fuente de energía absorbida, lo que corresponde de un 50 a $70 \%$ de la energía digestible (Zhang, 2017). Se reporta que del 6 al $10 \%$ de la energía bruta consumida por una vaca se convierte a metano, y se elimina por vía respiratoria (Eckard et al., 2010). El exceso de dihidrógeno $\left(\mathrm{H}_{2}\right)$ generado durante la conversión de glucosa a ácido acético o butírico, y el $\mathrm{CO}_{2}$ producido por la descarboxilación de metabolitos durante la fermentación, son utilizados por arqueas metanogénicas; las cuales los utilizan como sustratos para formar metano (Alayón et al., 2018). Cabe resaltar la relevancia de los protozoos en los procesos de formación de metano, pues ellos mantienen una relación simbiótica con las arqueas metanogénicas, con quienes están asociados intra y extracelularmente y realizar la transferencia de este hidrógeno (Hook et al., 2010).

Existen metodologías usadas para reducir la cantidad de $\mathrm{CH}_{4}$ producido por los rumiantes; entre ellas la implementación de aditivos químicos en la alimentación del ganado; sin embargo, el uso de estos se ha visto limitado por la aparición residual en los productos de consumo humano, lo que originó su prohibición en algunas legislaciones, como la de la Unión Europea en enero de 2006 (Alejos et al., 2018). Estas medidas dieron origen a la búsqueda de nuevas estrategias y alternativas orientadas al uso de aditivos naturales, como los extractos de plantas; los cuales son una opción en la alimentación del ganado para modificar su fermentación ruminal de manera positiva y mitigar las emisiones de $\mathrm{CH}_{4}$ entérico (Wallace, 2004).

Por ello, las prácticas orientadas a la mitigación de metano, pueden constituirse en factores importantes que impactan en la rentabilidad de un sistema de producción (Alayón et al., 2018). En este sentido, es importante considerar que cualquier práctica de mitigación que requiera una inversión adicional y que no sea compensada por agentes externos, o que afecte a la productividad animal, o aumente los costos de producción; es muy probable que se ha rechazado por el productor (Gerber et al., 2013).

Una alternativa de mitigación para evitar incrementos en la producción de metano, es el uso de extractos de plantas; los cuales contienen compuestos bioactivos que han sido investigados como alternativa en nutrición animal, cambiando la degradabilidad y fermentación del alimento; este efecto está relacionado con su actividad química y/o propiedades antimicrobianas de los extractos (Patra y Saxena, 2010). Por lo tanto, el objetivo fue obtener y caracterizar los extractos de plantas (Larrea tridentata, Origanum vulgare, Artemisa ludoviciana y Ruta graveolens), para probarlas en la digestibilidad ruminal in vitro y cuantificar la producción de gas, AGV y producción de metano. 


\section{MATERIAL Y MÉTODOS}

\section{Región de estudio y recolección de muestras}

El estudio se llevó a cabo en el estado de Zacatecas, México, se utilizaron plantas (hojas y flor) de Larrea tridentata, Origanum vulgare, Artemisa ludoviciana y Ruta graveolens; éstas fueron recolectadas de crecimiento silvestre durante primavera-verano de 2014 y 2015 de manera aleatoria. Cada una de las plantas fue secada a temperatura ambiente durante dos semanas, posteriormente desecadas a $45{ }^{\circ} \mathrm{C}$ en un horno convencional durante $24 \mathrm{~h}$ para eliminar la humedad; se molieron y almacenaron en bolsas de plástico.

\section{Obtención de los extractos de plantas}

En la extracción se utilizó $20 \mathrm{~g}$ de la muestra molida por cada $300 \mathrm{ml}$ de etanol, al $70 \%$ (J.T. Baker); fue colocada en frascos color ámbar. La mezcla se selló y agitó vigorosamente por $10 \mathrm{~min}$, se dejó macerar durante un mes a temperatura ambiente. El sobrenadante se filtró (Whatman No. 2), para remover los restos del polvo de la planta y el solvente fue evaporado en un extractor tipo Soxhlet a $85^{\circ} \mathrm{C}$. Se hicieron dos alícuotas; una de ellas fue utilizada para determinar la composición química del extracto y la otra para evaluar la actividad antimicrobiana.

\section{Composición química del extracto por cromatografía de gases}

Se determinó mediante un cromatógrafo de gases (CG; Agilent Tecnologies serie 6890N), empleando la columna polar DB_WAXetr. Las condiciones de trabajo fueron: temperatura después de la inyección $250^{\circ} \mathrm{C}$, a una presión de 12.13 psi con un flujo de He $36.5 \mathrm{ml}$ $\mathrm{min}^{-1}$. Las condiciones para la columna fueron: temperatura inicial $50^{\circ} \mathrm{C}$, de cero a dos minutos, con un aumento de $10^{\circ} \mathrm{C}$ hasta llegar a $250^{\circ} \mathrm{C}$; manteniendo esta temperatura constante por $5 \mathrm{~min}$, para luego descender a $50{ }^{\circ} \mathrm{C}$ por dos minutos, con un flujo de $\mathrm{He}$ de $1.6 \mathrm{~mL} \mathrm{~min}^{-1}$ a una presión de $12.13 \mathrm{psi}$ y una velocidad promedio de $25 \mathrm{~cm} \mathrm{~s}^{-1}$. Utilizando un detector de flama ionizante (FID) a una temperatura de $210^{\circ} \mathrm{C}$, con un flujo de $\mathrm{H}_{2}$ de $40 \mathrm{ml} \mathrm{min}^{-1}$ y un flujo de aire de $450 \mathrm{ml} \mathrm{min}^{-1}$. Previamente se realizó una curva de calibración, los estándares utilizados fueron: grado reactivo, marca Sigma Aldrich: carvacrol, timol, limoneno, linalol y terpineno, con un porcentaje de pureza de 98, 99.5, 98,97 y 85 \% respectivamente. Cada una de las determinaciones se realizó por triplicado.

\section{Determinación de la producción de gas in vitro}

Alimentación de los ovinos

Para la producción de gas in vitro se utilizó fluido ruminal de dos ovinos de pelo, canulados, alimentados con una dieta que contenía $83 \%$ de heno (50\% de alfalfa y 50 $\%$ de paja de trigo) y $17 \%$ de concentrado ( $63 \%$ maíz molido, $25 \%$ de harinolina, $5.5 \%$ de carbonato de calcio, $5.5 \%$ de fosfato mono-cálcico, $0.5 \%$ de pre mezcla de vitaminas A, D y E, $0.5 \%$ de microminarales) (NRC, 2003). El alimento se proporcionó diariamente 
a las 08:00 y 16:00 horas, con acceso libre al agua. Se alimentó a los ovinos por 30 días antes de la extracción del fluido ruminal, como tiempo de adaptación a la ración.

Producción de gas in vitro

Los extractos fueron adicionados de manera individual en cada una de las jarras de digestibilidad, en diferente volumen (Agarwal et al., 2009); como control se utilizó el sustrato de alfalfa sin la adición de aditivos. La producción de gas in vitro se determinó empleando el método propuesto por Theodorou et al. (1994); para lo cual se utilizaron unidades de fermentación (UF) de $120 \mathrm{ml}$ para cada muestra. En el registro de gas producido, se utilizó un medidor de presión marca Sper Scientific; la presión de gas fue acumulativa y determinada en unidades de presión (Psi); el tiempo de medición fue a las $3,6,9,12,24$ y $48 \mathrm{~h}$. Para cada volumen de los diferentes extractos, se realizaron tres repeticiones.

\section{Determinación de ácidos grasos volátiles en líquido ruminal}

Los AGVs (acético, propiónico y butírico) se cuantificaron por cromatografía de gases. Las condiciones de trabajo fueron: temperatura de entrada después de la inyección de la muestra es de $50{ }^{\circ} \mathrm{C}$ a una presión de $12.13 \mathrm{psi}$, con un flujo de He $36.5 \mathrm{ml} \mathrm{min}^{-1}$. Las condiciones para la columna fueron: temperatura inicial $50^{\circ} \mathrm{C}$, de cero a dos minutos con un aumento de $10^{\circ} \mathrm{C}$ por minuto hasta llegar a $250^{\circ} \mathrm{C}$, manteniendo esta temperatura constante por 5 minutos, para luego descender a $50{ }^{\circ} \mathrm{C}$ manteniendo por dos minutos

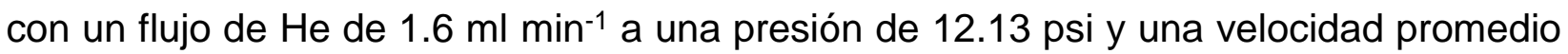
de $25 \mathrm{~cm} \mathrm{~s}^{-1}$.

Se utilizó un detector de flama ionizante (FID) a una temperatura de $210^{\circ} \mathrm{C}$, con un flujo de $\mathrm{H}_{2}$ de $40 \mathrm{ml} \mathrm{min}^{-1} \mathrm{y}$ un flujo de aire de $450 \mathrm{ml} \mathrm{min}^{-1}$. Previamente se realizó una curva de calibración. Los estándares utilizados fueron: grado reactivo, marca Sigma Aldrich: acético, propiónico y butírico; con un porcentaje de pureza de 99.5, 98 y $99 \%$ respectivamente. Cada una de las determinaciones se realizó por triplicado.

\section{Determinación de metano}

El metano se infirió a partir de la concentración de AGV, mediante la aplicación de modelos matemáticos no lineales establecido por Moss et al. (2000); donde se señala que la producción de $\mathrm{CH}_{4}$ se puede calcular de forma estequiométrica, empleando la siguiente ecuación:

$$
\left.\mathrm{CH}_{4}=0.45 C_{2}(\text { acetato })-0.275 \text { (propionato }\right)+0.4 C_{4}(\text { butirato })
$$

\section{Análisis estadístico}

Se utilizó un diseño completamente al azar para determinar diferencias entre los tratamientos y la prueba Tukey, para encontrar la diferencia entre los tratamientos con el paquete estadístico SPSS $\circledast$. 


\section{RESULTADOS Y DISCUSIÓN}

Los resultados en rendimiento de los extractos $L$. tridentata, $O$. vulgaris, A. Iudiviciana y $R$. graveolans (tabla 1), se deben al tiempo de concentración de la muestra en el equipo Soxleth, obteniendo el volumen del extracto $(\mathrm{ml})$, equivalente al rendimiento en porcentaje ( $\mathrm{w} / \mathrm{w})$, esto depende según el tiempo y modo de proceso, desde su recolección hasta su extracción. Los rendimientos de los extractos uno y dos de L. tridentata fueron de $11.6 \%$ y $30 \%$ respectivamente, esto se debe a el tiempo de concentración en el equipo Soxleth.

Tabla 1. Rendimientos por cada $20 \mathrm{~g}$ de muestra

\begin{tabular}{cccc}
\hline \hline Muestra & Extracto & $\begin{array}{c}\text { Volumen extracto } \\
(\mathbf{m L})\end{array}$ & $\begin{array}{c}\text { Rendimiento \% } \\
(\mathbf{w} / \mathbf{w})\end{array}$ \\
\hline \hline 1 & L. tridentata (2014) & 35 & 11.66 \\
2 & L. tridentata diluido (2015) & 90 & 30 \\
3 & O. vulgare (2014) & 41.5 & 13.83 \\
4 & O. vulgare (2015) & 30 & 10 \\
5 & A. ludoviciana (2014) & 36 & 12 \\
6 & A. ludoviciana diluido (2015) & 105 & 35 \\
7 & R. graveolens (2014) & 31.5 & 10.5 \\
\hline \hline
\end{tabular}

Para los extractos de $O$ vulgaris, los rendimientos fueron de $13.8 \%$ y $10 \%$. Albado et al. (2001), informan diferentes rendimientos según el tiempo y modo de proceso, desde su recolección hasta su extracción. Para $A$. ludiviciana el mayor rendimiento fue en el extracto seis, con un $35 \%$, y el menor en el extracto cinco con un $12 \%$. Por último, el rendimiento del extracto de $R$. graveolans fue de $10.5 \%$.

Los extractos preparados se analizaron por cromatografía de gases para conocer su composición química. El tiempo de corrimiento de los estándares en el CG fue de 18 min. con un tiempo de retención para terpineno de 3.524 min., limoneno 3.62 min., linalol 4.218 min., timol 8.870 min. y carvacrol 9.456 min. El volumen de inyección tanto para estándares como para muestras fue de $2 \mu \mathrm{L}$. En la tabla 2 se muestran las concentraciones empleadas de los estándares para la determinación de la concentración en las muestras, lo que permitió realizar una curva de calibración de la señal medida en función de la concentración del análito. La calibración permitió estimar los parámetros para determinar la linealidad de esa curva, y en consecuencia la capacidad de un método analítico para obtener resultados, que fueran directamente proporcionales a la concentración del compuesto en la muestra. En la tabla 3 se muestra la composición química de los extractos de L. tridentata $O$. vulgare, A. ludoviciana y $R$. graveolens.

Para la composición química se determinaron carvacrol, timol, terpineno, linalool y limoneno. En los extractos uno, dos y tres de L. tridentata se encontraron los compuestos timol y carvacrol en concentraciones para timol de $4.30 \mathrm{mg} \mathrm{ml}^{-1}$ y $0.30 \mathrm{mg} \mathrm{ml}^{-1}$ 
respectivamente, obtenidos mediante el uso de la ecuación de la regresión lineal de los estándares utilizados. Mientras que carvacrol las concentraciones fueron $7.79 \mathrm{mg} \mathrm{ml}^{-1} \mathrm{y}$ $4.43 \mathrm{mg} \mathrm{ml}^{-1}$, siendo mayor la concentración de este compuesto. Partes de las plantas medicinales (raíces, hojas, ramas, cortezas/tallos, flores y frutos) son comúnmente ricos en terpenos (carvacrol, citral, linalool y geraniol) y compuestos fenólicos (flavonoides y ácidos fenólicos), y estos han sido eficaces como aditivos alimentarios (Cai et al., 2004).

Tabla 2. Concentraciones de estándares empleadas para la determinación química de extractos de Larrea tridentata, Origanum vulgare, Artemisa ludoviciana y Ruta graveolens mediante cromatografía de gases.

\begin{tabular}{|c|c|c|c|c|c|}
\hline Estándar & $\begin{array}{c}\text { Timol } \\
(\mathrm{mg} \mathrm{ml}-1)\end{array}$ & $\begin{array}{l}\text { Carvacrol } \\
\text { (mg ml-1) }\end{array}$ & $\begin{array}{c}\text { Linalol } \\
\text { (mg ml-1) }\end{array}$ & $\begin{array}{l}\text { Terpineno } \\
\text { (mg ml-1) }\end{array}$ & $\begin{array}{r}\text { Limoneno } \\
\text { (mg ml-1) }\end{array}$ \\
\hline $\bar{~} 1$ & 10.37 & 8.28 & 7.74 & 7.15 & 8.49 \\
\hline 2 & 5.18 & 4.14 & 3.87 & 3.57 & 4.24 \\
\hline 3 & 2.59 & 2.07 & 1.93 & 1.78 & 2.12 \\
\hline 4 & 1.29 & 1.03 & 0.96 & 0.89 & 1.06 \\
\hline 5 & 0.64 & 0.51 & 0.48 & 0.44 & 0.53 \\
\hline 6 & 0.32 & 0.25 & 0.24 & 0.22 & 0.26 \\
\hline
\end{tabular}

Tabla 3. Composición química por cromatografía de gases

\begin{tabular}{clccccc}
\hline \hline Muestra Extracto & $\begin{array}{c}\text { Terpineno } \\
\left(\mathbf{m g ~ m}^{-1}\right)\end{array}$ & $\begin{array}{c}\text { Limoneno } \\
\left(\mathbf{m g ~ m L}^{-1}\right)\end{array}$ & $\begin{array}{c}\text { Linalool } \\
\left(\mathbf{m g ~ m}^{-1}\right)\end{array}$ & $\begin{array}{c}\text { Timol } \\
\left(\mathbf{m g ~ m}^{-1}\right)\end{array}$ & $\begin{array}{c}\text { Carvacrol } \\
\left(\mathbf{m g ~ m}^{-1}\right)\end{array}$ \\
\hline \hline 1 & L. tridentata (2014) & 0 & 0 & 0 & 4.303 & 7.798 \\
2 & L. tridentata diluido (2015) & 0 & 0 & 0 & 0.302 & 4.434 \\
3 & O. vulgare (2014) & 0 & 0.066 & 0.125 & 4.185 & 9.102 \\
4 & O. vulgare (2015) & 0.063 & 0 & 0.100 & 4.360 & 10.750 \\
5 & A. ludoviciana (2014) & 0.051 & 0 & 0.288 & 0.025 & 0.049 \\
6 & A. ludoviciana diluido (2015) & 0 & 0 & 0.116 & 0.013 & 0.023 \\
7 & R. graveolens (2014) & 0 & 0.060 & 0 & 0 & 0 \\
\hline \hline
\end{tabular}

En los extractos de $O$. vulgaris se encontró que el extracto tres, entre su composición está el limoneno $\left(0.6 \mathrm{mg} \mathrm{ml}^{-1}\right)$, linalool $\left(0.125 \mathrm{mg} \mathrm{ml}^{-1}\right)$, timol $\left(4.18 \mathrm{mg} \mathrm{ml}^{-1}\right)$ y carvacrol $\left(9.10 \mathrm{mg} \mathrm{ml}^{-1}\right)$; el extracto cuatro se encontró terpineno a una concentración de $0.063 \mathrm{mg}$ $\mathrm{ml}^{-1}$, pero no limoneno. Albado et al. (2001), reporta en aceites de orégano la presencia de terpineoles, fenoles y compuestos relacionados metabólicamente con el carvacrol al $9 \%$ y $12.19 \%$ de terpineno.

En cuanto a la composición química de los extractos de $A$. ludoviciana, se encontró el extracto cinco: terpineno $\left(0.051 \mathrm{mg} \mathrm{ml}^{-1}\right)$, linalool $\left(0.288 \mathrm{mg} \mathrm{ml}^{-1}\right)$, timol $\left(0.025 \mathrm{mg} \mathrm{ml}^{-1}\right) \mathrm{y}$ carvacrol $\left(0.049 \mathrm{mg} \mathrm{ml}^{-1}\right)$. Para el extracto seis los compuestos fueron linalool $(0.116 \mathrm{mg}$ $\left.\mathrm{ml}^{-1}\right)$, timol $\left(0.013 \mathrm{mg} \mathrm{ml}^{-1}\right)$ y carvacrol $\left(0.023 \mathrm{mg} \mathrm{ml}^{-1}\right)$. Kordali et al. (2005) investigaron la composición química de $A$. ludoviciana, encontraron que el extracto contiene principalmente anetol (81\%), beta-ocimeno (6.5\%), limoneno (3.0\%) y metileugenol (1.8 
$\%)$; ninguno de estos compuestos reportados fue identificados en el presente trabajo. En el extracto de $R$. graveolans se encontró limoneno. De Feo et al. (2002) reportan que los terpenoides constituyen $11.2 \%$ del aceite de $R$. graveolans con a-pineno (1.3\%), limoneno (3.0\%) y cineole (2.9\%), como los principales constituyentes de monoterpenos. Los resultados coinciden con los autores en la determinación de limoneno, más no en la concentración.

En la figura 1 se observa la cinética de degradación ruminal aplicando para la modificación de la fermentación de extractos de las plantas $L$. tridentata $O$. vulgare, $A$. ludoviciana y $R$. graveolens. En la técnica de producción de gas in vitro se observó en el extracto de $L$. tridentata a $100 \mu \mathrm{L}$ mayor producción de gas en el extracto uno $(168.9 \mathrm{ml}$ $\mathrm{g}^{-1}$ ) (figura 1); pero conforme aumentó la concentración del extracto fue disminuyendo, siendo a $665 \mu \mathrm{L}$ de $50 \mathrm{ml} \mathrm{g}^{-1}$ (figura 3).

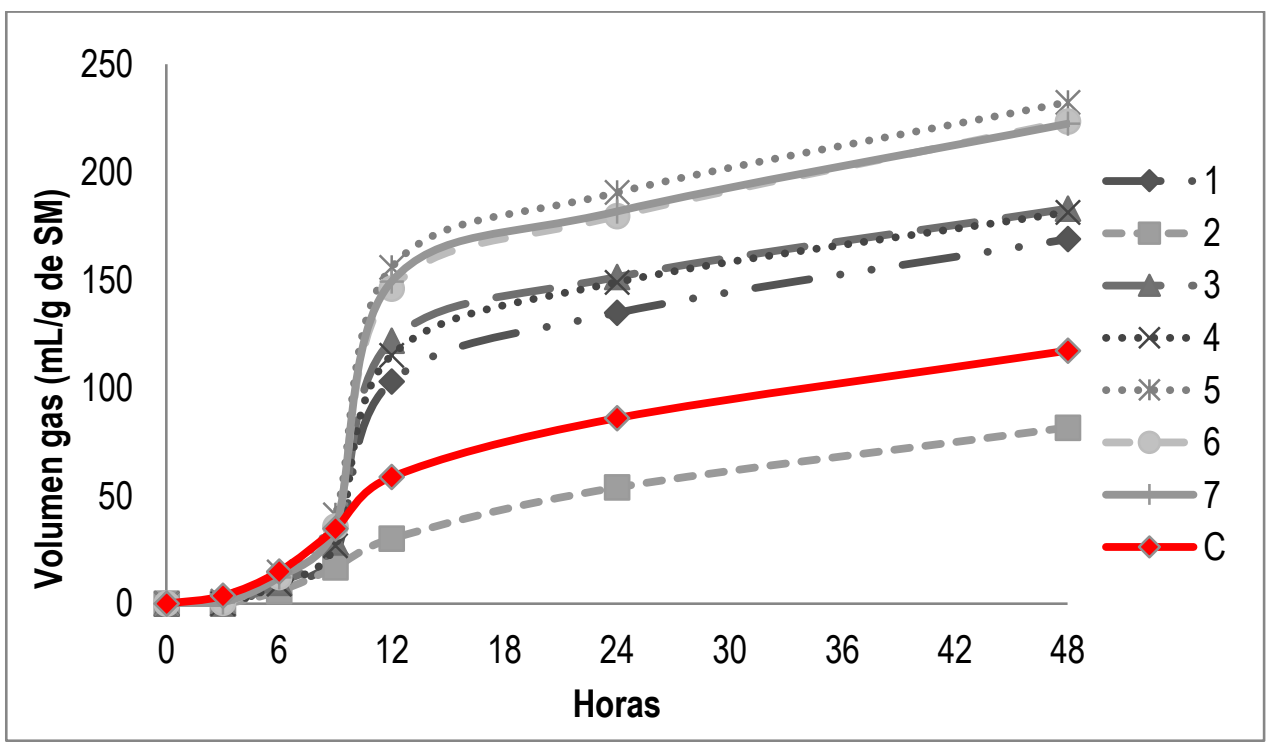

Figura 1. Digestibilidades de extractos a una dosis de $100 \mu \mathrm{L} \mathrm{dL}^{-1}$ de líquido ruminal.

En las digestibilidades para los extractos de 0 . vulgaris a $100 \mu \mathrm{L}$, la mayor producción de gas se reporta en el extracto tres $\left(98.54 \mathrm{ml} \mathrm{g}^{-1}\right)$, pero conforme aumentó la concentración del extracto también se incrementó la producción de gas, a $665 \mu \mathrm{L}$ de $180.06 \mathrm{ml} \mathrm{g}^{-1}$. Para el extracto cuatro la producción de gas fue $(665 \mu \mathrm{L})$, de $181.41 \mathrm{ml} \mathrm{g}^{-1}$. Para las digestibilidades de los extractos de A. Iudoviciana a $100 \mu \mathrm{L}, 330 \mu \mathrm{L}$ (figura 2) y $665 \mu \mathrm{L}$ en producción de gas en los extractos cinco y seis fue muy similar $232 \pm 27 \mathrm{ml} \mathrm{g}^{-1}$; en estas digestibilidades no se ve un cambio si se adiciona más o menos volumen. Por último, el extracto siete de $R$. graveolans reporta a $100 \mu \mathrm{L}$ un volumen de $222.39 \mathrm{ml} \mathrm{g}^{-1}$, a $330 \mu \mathrm{L}$ $219.19 \mathrm{ml} \mathrm{g}^{-1}$ y a $665 \mu \mathrm{L} 210.86 \mathrm{ml} \mathrm{g}^{-1}$ siendo mayor que el control de alfalfa empleado $\left(117.23 \mathrm{ml} \mathrm{g}^{-1}\right)$. 


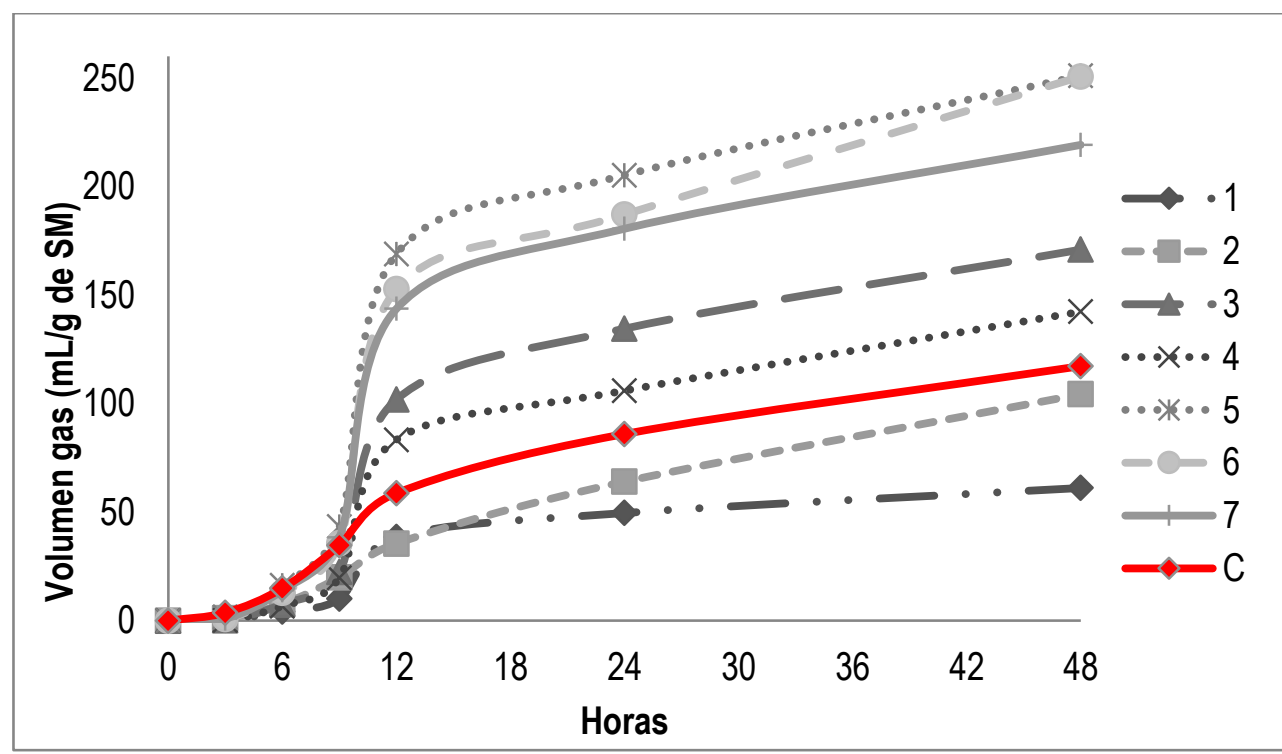

Figura 2. Digestibilidades de extractos a una dosis de $330 \mu \mathrm{L} \mathrm{dL}^{-1}$ de líquido ruminal.

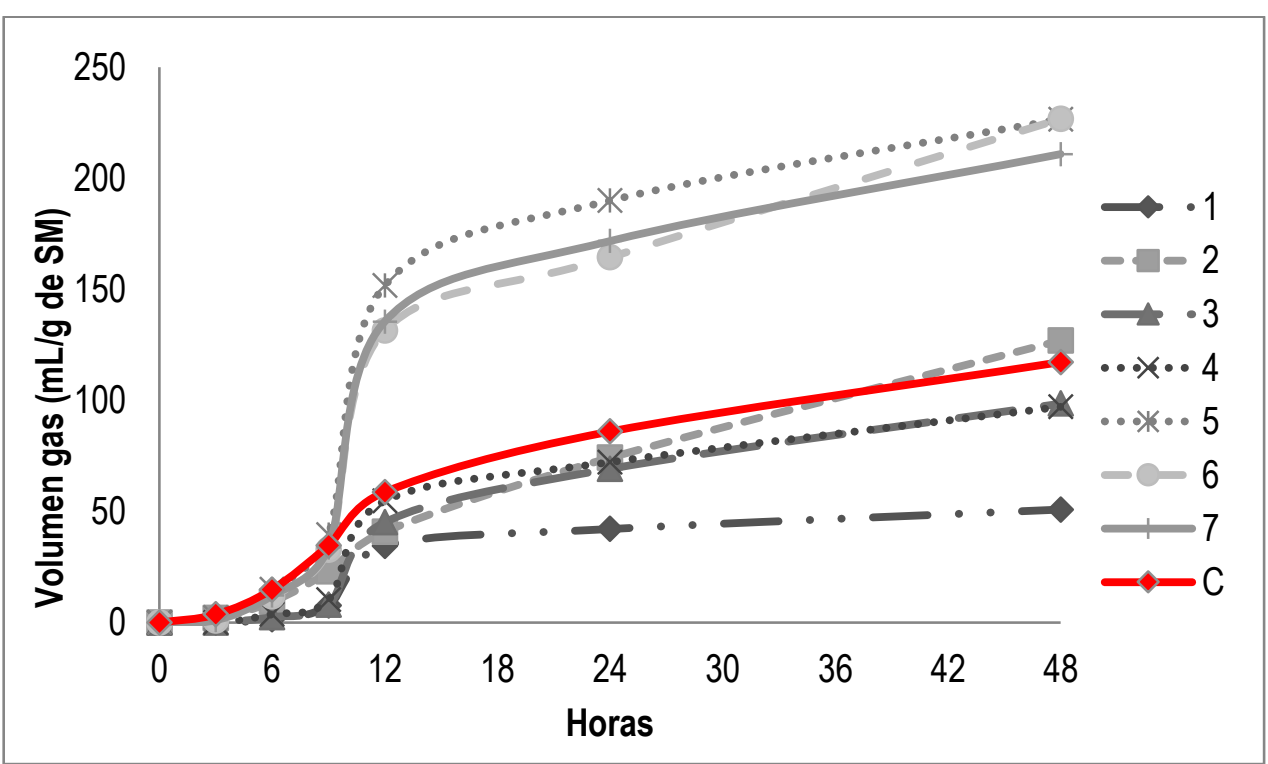

Figura 3. Digestibilidades de extractos a una dosis de $665 \mu \mathrm{L} \mathrm{dL}^{-1}$ de líquido ruminal.

En la tabla 4 se puede observar las medias para los grupos en los subconjuntos homogéneos y las diferencias significativas que existen entre los diferentes extractos y las dosis aplicadas de cada uno de ellos; confirmando de forma estadística que el gas $(\mathrm{mg} / \mathrm{ml})$ que se produjo, mostró diferencia estadísticamente significativa $(\mathrm{p}<0.05)$, ya que la mayor producción de gas se obtuvo en la dosis de extracto $(330 \mu \mathrm{L})$ de A. ludoviciana. Los compuestos secundarios de plantas, son reconocidos como agentes antimicrobianos que actúan contra las bacterias, protozoos y hongos. Los compuestos fenólicos son los principales componentes activos (Dorman y Deans, 2000, Burt, 2004), y la actividad 
antibacteriana se puede dar por una variedad de sustancias no fenólicas (Newton y Van 2002; Burt, 2004).

Tabla 4. Análisis de medias de la producción de gas con las diferentes dosis de los siete extractos utilizados.

\begin{tabular}{|c|c|c|c|c|c|c|c|}
\hline \multirow{2}{*}{$\begin{array}{c}\text { Extract } \\
0\end{array}$} & \multirow{2}{*}{$\begin{array}{c}\text { Dosis } \\
\text { de } \\
\text { extract } \\
o(\mu L)\end{array}$} & \multicolumn{6}{|c|}{ Gas producido $(\mathrm{mL} / \mathrm{g}) \pm{ }^{*} \mathrm{DE}$} \\
\hline & & 3 horas & 5 horas & 9 horas & 12 horas & 24 horas & 48 horas \\
\hline 1 & 100 & $0.10 \pm 0.12^{\mathrm{a}}$ & $8.49 \pm 0.74^{b}$ & $\begin{array}{r}23.08 \pm 1.59 \\
b\end{array}$ & $\begin{array}{r}102.88 \pm 7.35 \\
b\end{array}$ & $134.75 \pm 10^{b}$ & 168 \\
\hline 2 & 100 & $0.00 \pm 0.00^{\mathrm{a}}$ & $5.91 \pm$ & $\begin{array}{r}16.91 \pm 0.63 \\
\underline{a}\end{array}$ & $30.14 \pm 0.71^{a}$ & $53.85 \pm 0.68^{a}$ & $81.62 \pm 0.69^{a}$ \\
\hline 3 & 100 & $\begin{array}{r}0.23 \pm 0.10^{a} \\
b\end{array}$ & $9.78 \pm 0.30^{b}$ & $28.44 \pm 0.84^{c}$ & $121.86 \pm 2.29 c$ & $151.36 \pm 2.39$ & $183.07 \pm 2.28$ \\
\hline 4 & 100 & $0.00 \pm 0^{\mathrm{a}}$ & $9.18 \pm 0.28^{b}$ & $26.8 \pm 1.07^{b c}$ & $115.12 \pm 0.6^{b c}$ & $148.97 \pm 0.43$ & $181.42 \pm 2.89$ \\
\hline 5 & 100 & $1.18 \pm$ & $\begin{array}{r}14.67 \pm 0.93 \\
d\end{array}$ & $\begin{array}{r}41.22 \pm 1.99 \\
\mathrm{e}\end{array}$ & $\begin{array}{r}155.90 \pm 10.4 \\
d\end{array}$ & $3 \pm 11.7^{c}$ & $232.41 \pm 17.6^{c}$ \\
\hline 6 & 100 & $\begin{array}{r}0.45 \pm 0.45^{\mathrm{a}} \\
\mathrm{b}\end{array}$ & $12.48 \pm 0.95^{c}$ & $35.69 \pm 2.20$ & $145.93 \pm 8.92$ & $179.67 \pm 12.9^{c}$ & $223.46 \pm 19.4^{c}$ \\
\hline 7 & 100 & $0.65 \pm 0.29 \mathrm{bc}$ & $12.25 \pm 1.18^{c}$ & $\begin{array}{r}35.08 \pm 2.50 \\
d\end{array}$ & $\begin{array}{r}149.30 \pm 6.98 \\
d\end{array}$ & $181.77 \pm 10.3^{c}$ & $222.40 \pm 20.4^{c}$ \\
\hline 1 & 330 & $0.28 \pm 0.43^{\underline{a}}$ & $4.22 \pm 0.43^{\mathrm{a}}$ & $\begin{array}{r}10.02 \pm 0.69 \\
\underline{a}\end{array}$ & $38.42 \pm 2.94^{\mathrm{a}}$ & $49.56 \pm 3.23^{\mathrm{a}}$ & $61.10 \pm 2.90^{\mathrm{a}}$ \\
\hline 2 & 330 & $0.28 \pm 0.43^{\mathrm{a}}$ & $4.22 \pm 0.43^{\mathrm{a}}$ & $\begin{array}{r}10.02 \pm 0.69 \\
\underline{a}\end{array}$ & $38.42 \pm 2.94^{\mathrm{a}}$ & $\begin{array}{r}134.50 \pm 6.33 \\
\underline{a}\end{array}$ & $61.10 \pm 2.90^{\mathrm{a}}$ \\
\hline 3 & 330 & $0.19 \pm 0.19^{a}$ & $7.61 \pm 0.50^{b}$ & $\begin{array}{r}22.46 \pm 1.54 \\
b\end{array}$ & $101.87 \pm 6.52$ & $4 \pm 1.16^{c}$ & $170.94 \pm 7.59$ \\
\hline 4 & 330 & $0.08 \pm 0.10^{a}$ & $6.69 \pm 0.36^{a b}$ & $\begin{array}{r}19.78 \pm 0.71 \\
b\end{array}$ & $83.32 \pm 0.89^{b}$ & $\begin{array}{r}205.23 \pm 7.36 \\
b\end{array}$ & $\begin{array}{r}142.32 \pm 0.44 \\
\mathrm{~b}\end{array}$ \\
\hline 5 & 330 & $1.62 \pm 0.09 \mathrm{~b}$ & $\begin{array}{r}16.19 \pm 0.29 \\
d\end{array}$ & $\begin{array}{r}43.25 \pm 0.78 \\
d\end{array}$ & $\begin{array}{r}168.97 \pm 5.87 \\
d\end{array}$ & $\begin{array}{r}187.11 \pm 17.8 \\
\mathrm{e}\end{array}$ & $251.01 \pm 4.87$ \\
\hline 6 & 330 & $\begin{array}{r}0.63 \pm 0.55^{a} \\
b\end{array}$ & $12.71 \pm 0.99 c$ & $35.71 \pm 1.86^{c}$ & $152.67 \pm 19^{c d}$ & -4 de & $\begin{array}{r}250.71 \pm 16.2 \\
\mathrm{e}\end{array}$ \\
\hline 7 & 330 & $\begin{array}{r}0.89 \pm 0.80^{a} \\
b \\
\end{array}$ & $13.98 \pm 2.8^{\mathrm{cd}}$ & $38.40 \pm 5.3^{\mathrm{cd}}$ & $143.68 \pm 3.05^{c}$ & $\begin{array}{r}130.35 \pm 61.2 \\
\mathrm{e} \\
\end{array}$ & $\begin{array}{r}219.19 \pm 5.16 \\
\mathrm{e} \\
\end{array}$ \\
\hline 1 & & & & & $1.48^{\mathrm{a}}$ & & \\
\hline 2 & 665 & $04 \pm 0.05^{\mathrm{cd}}$ & & & $40.94 \pm 0.36^{\mathrm{ab}}$ & & $126.94 \pm 1.92^{c}$ \\
\hline 3 & 665 & & & & $45.13 \pm 3.20^{b}$ & & $98.55 \pm 8.55^{b}$ \\
\hline A & 665 & $=0^{a}$ & $3.68 \pm 0.36^{b}$ & $10.2 \pm 0.64^{b}$ & $54.74 \pm 1.96^{c}$ & $72.20 \pm 2.07^{b}$ & $97.11 \pm 1.99^{b}$ \\
\hline 5 & 665 & $2.16 \pm 0.52^{d}$ & e & $39.5 \pm 1.71^{\mathrm{e}}$ & & $189.94 \pm 7.08^{c}$ & 6 \\
\hline 6 & 665 & $\begin{array}{r}0.96 \pm 0.29 a \\
b\end{array}$ & $\begin{array}{r}11.83 \pm 0.67 \\
d\end{array}$ & $32.8 \pm 1.25^{d}$ & $131.39 \pm 1.25$ & $164.40 \pm 2.55^{c}$ & $226.69 \pm 4.47$ \\
\hline 7 & 665 & $1.08 \pm 0.9^{b c}$ & $12.13 \pm 0.8^{d}$ & $33.6 \pm 0.67^{d}$ & $\begin{array}{r}135.46 \pm 6.13 \\
d\end{array}$ & $171.72 \pm 5.82^{c}$ & $210.87 \pm 6.03$ \\
\hline
\end{tabular}

${ }^{*} \mathrm{DE}$ : Desviación estándar, valores de medias con letras distintas en la misma columna y en la misma dosis difieren estadisticamente $(p<0.05)$.

El modo de acción antimicrobiana, se considera principalmente por el potencial en la membrana de la célula bacteriana al desintegrar las estructuras de ésta, que provoca la fuga de iones; sus efectos sobre la actividad de los microorganismos ruminales depende 
de las especies de plantas consumidas y de su composición química. Monoterpenos particularmente alcoholes monoterpenos y aldehídos, inhiben fuertemente el crecimiento y el metabolismo de los microbios del rumen; mientras que los hidrocarburos monoterpenos inhiben ligeramente $y$, a veces estimulan la actividad microbiana del rumen (Benchaar et al., 2008). Los efectos de estos compuestos son modulados por el pH del rumen, la dieta en la que se incluyen y por métodos de preparación y de extracción (Patra y Saxena, 2010; Patra y Yu, 2014).

Se ha reportado que los extractos y aceites de plantas inhiben a las bacterias productoras de nitrógeno amoniacal, decreciendo así la desamimación de los aminoácidos, principalmente en dietas que contienen cantidades no muy altas de proteína (Patra y Saxena, 2010). También se ha observado que el número total de bacterias viables no es afectado, es decir cambian solamente las proporciones de grupos bacterianos; pero en estudios in vitro donde se han incluido dosis altas, como por ejemplo $400 \mathrm{\mu g} \mathrm{ml}^{-1}$ de timol en comparación con las comúnmente reportadas en la literatura, sí provocan descenso en el número total de microorganismos (McKay y Blumberg, 2006). Las modificaciones en el total y proporción de los AGV impactan sobre los perfiles de producción de metano, sobre todo si reduce la proporción de acetato y se incrementa la de propionato; por lo tanto, cambios en estos repercutirán positiva o negativamente en la producción de metano.

En volumen de $100 \mu \mathrm{L}$ por cada $100 \mathrm{ml}$ de líquido ruminal, al final de la digestibilidad se tomó muestra para la determinación de AGV; los resultados se muestran en la tabla 5. Asimismo, se reporta la producción de metano obtenida por la ecuación estequiométrica; de Moss et al. (2000), encontrando la mayor producción en metano con el extracto tres de $O$. vulgaris con $1.162 \mathrm{~g} \mathrm{~L}^{-1}$, y la mínima en el extracto de A. ludoviciana $0.204 \mathrm{~g} \mathrm{~L}^{-1}$.

Para la muestra donde se agregó $300 \mu \mathrm{L}$ por cada $100 \mathrm{ml}$ de líquido ruminal, los resultados encontrados son los siguientes, la mayor digestibilidad se reporta en las muestras cinco y seis correspondientes al extracto de A. ludoviciana. En la tabla 5 se observa los resultados de los AGV y la producción de metano, siendo los extractos seis de $A$. ludoviciana $\left(1.030 \mathrm{~g} \mathrm{~L}^{-1}\right)$ y siete de $R$. graveolans $\left(1.029 \mathrm{~g} \mathrm{~L}^{-1}\right)$ los de mayor producción generados en esta digestibilidad. Para la dosis de $665 \mu \mathrm{L}$ los resultados obtenidos son los siguientes; mayor digestibilidad se reporta en las muestras cinco y seis correspondientes al extracto de $A$. ludoviciana. En la tabla 5 se observa las concentraciones de los AGV y la producción de metano, siendo mayor en el extracto cinco de $A$. Iudoviciana ( $\left.1.148 \mathrm{~g} \mathrm{~L}^{-1}\right)$ y la menor producción de metano se reporta en el extracto uno de $L$. tridentata generando $0.193 \mathrm{~g} \mathrm{~L}^{-1}$.

En cuanto a la producción de AGV, la menor producción de ácido acético se reportó en el extracto uno con $665 \mu \mathrm{L}$, siendo de $0.680 \mathrm{~g} \mathrm{~L}^{-1}$ (producción de gas $50 \mathrm{ml} \mathrm{g}^{-1}$ ); mientras 
Tabla 5. Producción de AGV y metano a las $48 \mathrm{~h}$ de la digestibilidad ruminal aplicando 100, 330 y $665 \mu \mathrm{L}$ de extracto

\begin{tabular}{|c|c|c|c|}
\hline & $100 \mu \mathrm{L}$ & $\begin{array}{c}\text { Ácido acético } \\
\mathrm{g} \mathrm{L}^{-1} \pm \mathrm{DE} \\
330 \mu \mathrm{L} \\
\end{array}$ & $665 \mu \mathrm{L}$ \\
\hline Control & 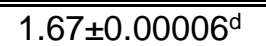 & $1.67 \pm 0.00006^{d}$ & $\bar{~} 1.67 \pm 0.00006^{b c}$ \\
\hline 1 & $1.72 \pm 0.00006^{e}$ & $0.90 \pm 0.00012^{a}$ & $0.6 \pm 0.00013^{a}$ \\
\hline 2 & $2.41 \pm 0.00002^{\mathrm{h}}$ & $1.73 \pm 0.00016^{e}$ & $1.83 \pm 0.00021^{c}$ \\
\hline 3 & $2.14 \pm 0.000249$ & $1.28 \pm 0.00017^{b}$ & $0.82 \pm 0.71442^{\mathrm{a}}$ \\
\hline 4 & $1.80 \pm 0.00007^{f}$ & $1.31 \pm 0.00009^{c}$ & $1.01 \pm 0.00009^{\mathrm{ab}}$ \\
\hline 5 & $0 \pm 0^{\mathrm{a}}$ & $1.74 \pm 0.00006^{f}$ & $1.99 \pm 0.00027^{c}$ \\
\hline 6 & $0.62 \pm 0.00013^{b}$ & $1.84 \pm 0.00026^{g}$ & $1.97 \pm 0.00009^{c}$ \\
\hline \multirow[t]{2}{*}{7} & $1.59 \pm 0.00007^{c}$ & $1.86 \pm 0.00005^{\mathrm{h}}$ & $1.94 \pm 0.00005^{c}$ \\
\hline & & Ácido propiónico & \\
\hline Control & $0.923 \pm 0.00017^{c}$ & $0.923 \pm 0.00017^{b}$ & $0.923 \pm 0.00017^{d}$ \\
\hline 1 & $0 \pm 0^{a}$ & $0 \pm 0^{a}$ & $0.436 \pm 0.00020^{b}$ \\
\hline 2 & $1.19 \pm 0.00016^{\mathrm{d}}$ & $0 \pm 0^{a}$ & $0 \pm 0^{a}$ \\
\hline 3 & $0 \pm 0^{\mathrm{a}}$ & $0 \pm 0^{a}$ & $0 \pm 0^{\mathrm{a}}$ \\
\hline 4 & $0 \pm 0^{\mathrm{a}}$ & $0.94 \pm 0.00017^{c}$ & $0 \pm 0^{\mathrm{a}}$ \\
\hline 5 & $0 \pm 0^{\mathrm{a}}$ & $0 \pm 0^{\mathrm{a}}$ & $0 \pm 0^{\mathrm{a}}$ \\
\hline 6 & $0 \pm 0^{\mathrm{a}}$ & $0 \pm 0^{\mathrm{a}}$ & $0 \pm 0^{\mathrm{a}}$ \\
\hline \multirow[t]{2}{*}{7} & $0.74 \pm 0.00018^{b}$ & $0 \pm 0^{a}$ & $0.883 \pm 0.00018^{c}$ \\
\hline & & Ácido butírico & \\
\hline Control & $0.036 \pm 0.00022^{b}$ & $0.037 \pm 0.00022^{b}$ & $0.037 \pm 0.00022^{\mathrm{a}}$ \\
\hline 1 & $0.434 \pm 0.00013^{e}$ & $0.37 \pm 0.00015^{d}$ & $0.016 \pm 0.00022^{a}$ \\
\hline 2 & $0.053 \pm 0.00021^{c}$ & $0.424 \pm 0.00014^{\mathrm{e}}$ & $0.437 \pm 0.00013^{b c}$ \\
\hline 3 & $0.492 \pm 0.00012 f$ & $0.349 \pm 0.00015^{c}$ & $0.413 \pm 0.15101^{b c}$ \\
\hline 4 & $0.539 \pm 0.00011^{\mathrm{h}}$ & $0.014 \pm 0.00022^{\mathrm{a}}$ & $0.336 \pm 0.00016^{b}$ \\
\hline 5 & $0.509 \pm 0.00012^{g}$ & $0.444 \pm 0.00013^{\dagger}$ & $0.622 \pm 0.00010^{d}$ \\
\hline 6 & $0.076 \pm 0.00021^{d}$ & $0.482 \pm 0.00012^{\mathrm{h}}$ & $0.508 \pm 0.00012^{\mathrm{cd}}$ \\
\hline \multirow[t]{2}{*}{7} & $0.029 \pm 0.00022^{\mathrm{a}}$ & $0.469 \pm 0.00013^{g}$ & $0.037 \pm 0.00022^{\mathrm{a}}$ \\
\hline & & Metano & \\
\hline Control & $0.514 \pm 0.00007^{c}$ & $0.515 \pm 0.00007^{b}$ & $0.515 \pm 0.00007^{b}$ \\
\hline 1 & $0.951 \pm 0.00008^{f}$ & $0.554 \pm 0.00011^{c}$ & $0.193 \pm 0.00009^{a}$ \\
\hline 2 & $0.777 \pm 0.00005^{\mathrm{e}}$ & $0.95 \pm 0.00012^{\mathrm{e}}$ & $1.002 \pm 0.00014^{c}$ \\
\hline 3 & $1.162 \pm 0.00010^{\mathrm{h}}$ & $0.716 \pm 0.00010^{d}$ & $0.536 \pm 0.26108^{b}$ \\
\hline 4 & $1.03 \pm 0.00007 \mathrm{~g}$ & $0.338 \pm 0.00008^{\mathrm{a}}$ & $0.59 \pm 0.00010^{\mathrm{b}}$ \\
\hline 5 & $0.204 \pm 0.00005^{\mathrm{a}}$ & $0.964 \pm 0.00008^{f}$ & $1.148 \pm 0.00011^{\mathrm{c}}$ \\
\hline 6 & $0.312 \pm 0.00014^{b}$ & $1.021 \pm 0.00016^{\mathrm{g}}$ & $1.09 \pm 0.00006^{c}$ \\
\hline 7 & $0.525 \pm 0.00007^{d}$ & $1.029 \pm 0.00007^{\mathrm{h}}$ & $0.648 \pm 0.00006^{b}$ \\
\hline
\end{tabular}

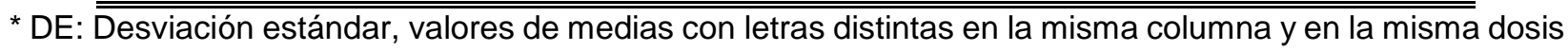
difieren estadisticamente $(p<0.05)$.

que el extracto dos presentó la mayor concentración a $100 \mu \mathrm{L}\left(2.411 \mathrm{~g} \mathrm{~L}^{-1}\right)$; siendo éste el que menor cantidad de gas produjo a esta concentración $\left(81.62 \mathrm{ml} \mathrm{g}^{-1}\right)$. Para ácido propiónico se inhibe la producción de este AGV, sólo encontrándose en el extracto dos a $100 \mu \mathrm{L}\left(1.197 \mathrm{~g} \mathrm{~L}^{-1}\right)$, y en el extracto uno a $665 \mu \mathrm{L}\left(0.436 \mathrm{~g} \mathrm{~L}^{-1}\right)$. El ácido butírico presentó concentración no mayor a $0.622 \mathrm{~g} \mathrm{~L}^{-1} 100 \mu \mathrm{L}, 330 \mu \mathrm{L}$ y $665 \mu \mathrm{L}$. En cuanto a la producción de metano calculada fue menor en el extracto uno a $330 \mu \mathrm{L}\left(0.554 \mathrm{~g} \mathrm{~L}^{-1}\right)$ y a $665 \mu \mathrm{L}(0.193$ $\left.\mathrm{g} \mathrm{L}^{-1}\right)$ con respecto a el estándar de alfalfa que produjo $0.514 \mathrm{~g} \mathrm{~L}^{-1}$ de metano; la mayor producción de metano se reporta a $665 \mu \mathrm{L}$ en el extracto dos $\left(1.002 \mathrm{~g} \mathrm{~L}^{-1}\right)$, siendo ésta la 
mayor producción de gas (126.94 $\left.\mathrm{ml} \mathrm{g}^{-1}\right)$ a este volumen de extracto. Agarwal et al. (2009) y Cobellis et al. (2016), reportan que al adicionar extractos y aceites de plantas en la producción de AGV, éstos se modifican; ellos reportan que la relación de acetatopropionato se incrementa; aunque en otros estudios no se han encontrado diferencias al adicionar extractos (Ravindra et al., 2009, Wang et al., 2009).

La menor producción de ácido acético se reportó en el extracto tres con $665 \mu \mathrm{L}$, siendo de $0.825 \mathrm{~g} \mathrm{~L}^{-1}$ (producción de gas $180.54 \mathrm{ml} \mathrm{g}^{-1}$ ); mientras que el extracto tres presentó la mayor concentración a $100 \mu \mathrm{L}\left(2.145 \mathrm{~g} \mathrm{~L}^{-1}\right)$, siendo éste uno de los que menos gas produjo $\left(98.54 \mathrm{ml} \mathrm{g}^{-1}\right)$.

Para ácido propiónico se inhibe la producción de este AGV, sólo encontrándose en el extracto cuatro a $330 \mu \mathrm{L}\left(0.947 \mathrm{~g} \mathrm{~L}^{-1}\right)$ y una producción de gas de $143.32 \mathrm{~mL} \mathrm{~g}^{-1}$. Mientras que el ácido butírico presentó la concentración mayor de $0.539 \mathrm{~g} \mathrm{~L}^{-1}$ a $100 \mu \mathrm{L}$, y la menor concentración se reporta en el extracto cuatro a $330 \mu \mathrm{L}\left(0.014 \mathrm{~g} \mathrm{~L}^{-1}\right)$. En cuanto a la producción de metano calculado fue menor en el extracto tres a $665 \mu \mathrm{L}\left(0.536 \mathrm{~g} \mathrm{~L}^{-1}\right)$, y en el extracto cuatro a $665 \mu \mathrm{L}\left(0.590 \mathrm{~g} \mathrm{~L}^{-1}\right)$, con respecto a el control de alfalfa que produjo $0.514 \mathrm{~g} \mathrm{~L}^{-1}$ de metano. La mayor producción de metano se reporta a $100 \mu \mathrm{L}$ en el extracto tres $\left(1.162 \mathrm{~g} \mathrm{~L}^{-1}\right)$, siendo ésta una de la menor producción de gas $\left(98.54 \mathrm{~mL} \mathrm{~g}^{-1}\right)$ a este volumen de extracto.

El extracto seis con $100 \mu \mathrm{L}$ presentó una concentración de ácido acético de $0.625 \mathrm{~g} \mathrm{~L}^{-1}$ (producción de gas $223.45 \mathrm{ml} \mathrm{g}^{-1}$ ), mientras que el extracto cinco presentó la mayor concentración a $665 \mu \mathrm{L}\left(1.997 \mathrm{~g} \mathrm{~L}^{-1}\right)$, con una producción de gas de $226.54 \mathrm{~mL} \mathrm{~g}^{-1}$. Para ácido propiónico se inhibe la producción de este AGV. Mientras que el ácido butírico presentó concentraciones no mayores a $0.746 \mathrm{~g} \mathrm{~L}^{-1}$ a $100 \mu \mathrm{L}, 330 \mu \mathrm{L}$ y $665 \mu$. La menor producción se observa a $100 \mu \mathrm{L}$ en el extracto seis $\left(0.076 \mathrm{~g} \mathrm{~L}^{-1}\right)$.

En cuanto a la producción de metano calculado, fue menor en el extracto cinco a $100 \mu \mathrm{L}$ $\left(0.204 \mathrm{~g} \mathrm{~L}^{-1}\right)$ y en el seis a $100 \mu \mathrm{L}\left(0.312 \mathrm{~g} \mathrm{~L}^{-1}\right)$, con respecto al control de alfalfa que produjo $0.514 \mathrm{~g} \mathrm{~L}^{-1}$ de metano. La mayor producción de metano se reporta a $665 \mu \mathrm{L}$ en el extracto cinco (1.148 $\left.\mathrm{g} \mathrm{L}^{-1}\right)$, siendo ésta la mayor producción de gas $\left(226.54 \mathrm{~mL} \mathrm{~g}^{-1}\right)$ a este volumen.

En el extracto de $R$. graveolans a $100 \mu \mathrm{L}$, la producción de ácido acético se reportó de $1.596 \mathrm{ml} \mathrm{g}^{-1}$ (producción de gas $222.39 \mathrm{ml} \mathrm{g}^{-1}$ ), a $330 \mu \mathrm{L}$ de $1.869 \mathrm{~g} \mathrm{~L}^{-1}$ (producción de gas $219.19 \mathrm{ml} \mathrm{g}^{-1}$ ) y a $665 \mu \mathrm{L}$ de $1.947 \mathrm{~mL} \mathrm{~g}^{-1}$ (producción de gas $210.86 \mathrm{ml} \mathrm{g}^{-1}$ ); mientras que el estándar de alfalfa reporta $0.923 \mathrm{ml} \mathrm{g}^{-1}$ siendo mayor la encontrada en el extracto siete a $330 \mu \mathrm{L}$ y a $665 \mu \mathrm{L}$. Para ácido propiónico la producción es a $100 \mu \mathrm{L}$ de $0.746 \mathrm{~g} \mathrm{~L}$ ${ }^{1}$ y a $665 \mu \mathrm{L}$ de $0.883 \mathrm{~g} \mathrm{~L}^{-1}$; mientras que el ácido butírico presentó la concentración mayor de $0.469 \mathrm{~g} \mathrm{~L}^{-1}$ a $330 \mu \mathrm{L}$; la menor producción se observa a $100 \mu \mathrm{L}$ de $0.029 \mathrm{~g} \mathrm{~L}^{-1}$. En cuanto a la producción de metano calculado, fue menor a $100 \mu \mathrm{L}\left(0.525 \mathrm{~g} \mathrm{~L}^{-1}\right)$ (siendo donde se produjo la mayor cantidad de gas $222.39 \mathrm{ml} \mathrm{g}^{-1}$ ) y a $330 \mu \mathrm{L}$ se produjo $1.029 \mathrm{~g}$ 
$\mathrm{L}^{-1}$; el estándar de alfalfa produjo $0.515 \mathrm{~g} \mathrm{~L}^{-1}$ de metano, lo que nos indica que no hubo disminución de éste con el extracto de $R$. graveolans.

Debido a que del 40 al $60 \%$ del total de los gases de efecto invernadero (GEI) de la ganadería provienen de la fermentación entérica, el manejo del estiércol y las diferentes actividades relacionadas con la obtención de alimentos para los animales (Sejian et al., 2015); una estrategia a seguir y considerar para la mitigación de metano se deberá enfocar a la modificación de la fermentación ruminal sin alterar la producción animal, mejorando la conversión del alimento.

\section{CONCLUSIÓN}

El extracto de $O$. vulgaris con mayor concentración de timol y carvacrol presentó mejor digestibilidad de gas, menor producción de AGV y menor concentración de metano empleando los volúmenes 330 y $665 \mu \mathrm{L}$; por lo tanto, consideramos necesario continuar investigando el empleo de los extractos estudiados como aditivos en pruebas in vivo.

\section{LITERATURA CITADA}

AGARWAL N, Shekhar C, Kumar R, Chaudhary L, Kamra DN. 2009. Effect of peppermint (Mentha piperita) oil on in vitro methanogenesis and fermentation of feed with buffalo rumen liquor. Animal Feed Science and Technology. 148: 321-327. Doi: 10.1016/j.anifeedsci.2008.04.004

ALAYÓN GJA, Jiménez FG, Piñeiro VAT, Canul SJ, Albores MS, Villanueva LG, Nahed TJ, Ku V. 2018. Estrategias de mitigación de gases de efecto invernadero en la ganadería. Agroproductividad. 11: 9-15. $\quad$ http://www.revistaagroproductividad.org/index.php/agroproductividad/article/view/112/100

ALEJOS FJI, Almaraz BI, Peralta OJJG, Sánchez SP, Soriano RR, Torres CMG. 2018. Consideraciones de bienestar animal en las estrategias para la disminución de la producción de metano entérico por rumiantes. Agroproductividad. 11: 57-63. ISSN: 24487546120

ALBADO PE, Sáez FG, Grabiel AS. 2001. Composición química y actividad antibacteriana del aceite esencial del Origanum vulgare (orégano). Medical Journal Herediana. 12(1): 16-19. ISSN: 1729-214X

BENCHAAR C, Calsamiglia S, Chaves AV, Fraser GR, Colombatto D, McAllister TA, Beauchemin KA. 2008. A review of plant-derived essential oils in ruminant nutrition and production. Animal Feed Science and Technology. 145(1-4): 209-228. Doi:10.1016/j.anifeedsci.2007.04.014 
BURT S. 2004. Essential oils: their antibacterial properties and potential applications in foods -a review. International Journal of Food Microbiology. 94(3): 223-253. Doi: 10.1016/j.ijfoodmicro.2004.03.022

CAI Y, Luo Q, Sol M, Corke H. 2004. Antioxidant activity and phenolic compounds of 112 traditional Chinese medicinal plants associated with anticancer. Life sciences. 74(17): 2157-2184. Doi: 10.1016/j.lfs.2003.09.047

COBELLIS G, Trabalza-Marinucci M, Yu Z. 2016. Critical evaluation of essential oils as rumen modifiers in ruminant nutrition: A review. Science of the Total Environment, 545, 556-568. Doi: 10.1016/j.scitotenv.2015.12.103

DE FEO V, De Simone F, Senatore F. 2002. Potential allelochemicals from the essential oil of Ruta graveolens. Phytochemistry. 61(5): 573-578. Doi: 10.1016/S00319422(02)00284-4

DORMAN HJD, Deans SG. 2000. Animicrobial agents from plants: antibacterial activity of plant volatile oils. Journal of applied microbiology. 88(2): 308-316. Doi: 10.1.1.838.5779\&rep=rep1\&type=pdf

ECKARD RJ, Grainger C, De Klein CAM. 2010. Options for the abatement of methane and nitrous oxide from ruminants' production: a review. Livestock Science. 130: 47-56. Doi: 10.1016/j.livsci.2010.02.010

FRANCE J. 2005. "Volatile fatty acid production". In Dijkstra J, Forbes JM, France J. Quantitative aspects of ruminant digestion and metabolism. 2nd edition. CABI Publishing. Pp. 157-175. USA. ISBN: 0851998143

GALICIA JMM, López GSJ, Ávila SNY, Murialdo SE. 2017. Two-component system: a molecular dialogue between ruminal bacteria and feed particles (forage plants). Tropical and Subtropical Agroecosystems. 20(3). ISSN: 1870-0462

GERBER PJ, Steinfeld H, Henderson B, Mottet A, Opio C, Dijkman J, Falcucci A, Tempio G. 2013. Tackling Climate Change Through Livestock-A Global Assessment of Emissions and Mitigation Opportunities. Rome, Italy: FAO-Food and Agriculture Organization of the United Nations. http://www.fao.org/.../i3437e00.htm

GETACHEW G, Robinson PH, DePeters EJ, Taylor SJ, Gisi DD, Higginbotham GE, Riordan TJ. 2005. Methane production from commercial dairy rations estimated using an in vitro gas technique. Animal Feed Science and Technology. 123: 391-402. Doi: 10.1016/j.anifeedsci.2005.04.056 
HOOK SE, Wright ADG, McBride BW. 2010. Methanogens: methane producers of the rumen and mitigation strategies. Archaea. 2010: 1-11. Doi: 10.1155/2010/945785

HRISTOV AN, Oh J, Giallongo F, Frederick TW, Harper MT, Weeks HL, Branco AF, Moate PJ, Deighton MH, Williams SR, Kindermann M, Duval S. 2015. An inhibitor persistently decreased enteric methane emission from dairy cows with no negative effect on milk production. Proceedings of the National Academy of Sciences. 112(34): 10663-10668. Doi: 10.1073/pnas.1504124112.

JOHNSON DE, Branine M, Ward GM, Carinean B, Lodman D. 1991. The potential contribution of beef cattle methane to total global warning: Background information and perspective. In: Beef program report. The Department of Animal Science's USA. p. 8. http://afcerc.tamu.edu/publications/Publication-PDFs

KORDALI S, Cakir A, Mavi A, Kilic H, Yildirim A. 2005. Screening of chemical composition and antifungal and antioxidant activities of the essential oils from three Turkish Artemisia species. Journal of agricultural and food chemistry. 53(3): 1408-1416. Doi: $10.1021 / \mathrm{jf0} 48429 \mathrm{n}$

MCKAY DL, Blumberg JB. 2006. A review of the bioactivity and potential health benefits of chamomile tea (Matricaria recutita L.). Phytotherapy research. 20(8): 619-633. Doi: 10.1002/ptr.1900

MOSS AR, Jouany JP, Newbold J. 2000. Methane production by ruminants: Its contribution to global warming. Annales de zootechnie. 49(3): 231-253. Doi: 10.1051/animres:2000119

MOUMEN A, Azizi G, Chekroun KB, Baghour M. 2016. The effects of livestock methane emission on the global warming: a review. International Journal of Global Warming. 9: 229-253. Doi: 10.1504/IJGW.2016.074956

NEWTON J, Van VK. 2002. Seasonal patterns and controlling factors of primary production in Puget Sound's central basin and Possession Sound, Washington State Department of Ecology, Environmental Assessment Program, Publication \#02-03-059, Olympia, WA. $38 \mathrm{p}$.

NRC (National Research Council). 2003. Air Emissions from Animal Feeding Operations: Current Knowledge, Future Needs (Natl Acad Press, Washington, DC). 286 p.

PATRA AK, Saxena J. 2010. A new perspective on the use of plant secondary metabolites to inhibit methanogenesis in the rumen. Phytochemistry. 71(11-12): 1198-1222. Doi: 10.1016/j.phytochem.2010.05.010 
PATRA AK, Yu Z. 2014. Effects of vanillin, quillaja saponin, and essential oils on in vitro fermentation and protein-degrading microorganisms of the rumen. Appl Microbiol Biotechnol. 98:897-905. Doi: 10. 1007/s00253-013-4930-x.

POPOVA M, McGovern E, McCabe MS, Martin C, Doreau M, Arbre M, Meale SJ, Morgavi DP, Waters, SM 2017. La capacidad estructural y funcional de la microbiota ruminal y cecal en el ganado en crecimiento no se vio afectada por la suplementación dietética de aceite de linaza y nitrato. Fronteras en microbiología, 8, 937. Doi: 10.3389/fmicb.2017.00937

RAVINDRA K, Kamra DN, Neeta A, Chaudhary LC. 2009. Effect of eucalyptus (Eucalyptus globules) oil on in vitro methanogenesis and fermentation of feed with buffalo rumen liquor. Animal Nutrition and Feed Technology. 9(2): 237-243. ISSN: 0972-2963

SEJIAN V, Bhatta R, Soren NM, Malik PK, Ravindra JP, Prasad CS, Lal R. 2015. Introduction to Concepts of Climate Change Impact on Livestock and Its Adaptation and Mitigation. Climate Change Impact on Livestock: Adaptation and Mitigation. Doi: 10.1007/978-81-322-2265-1_1, Springer India, p 1-25.

TAPIO I, Snelling TJ, Strozzi F, Wallace RJ. 2017. The ruminal microbiome associated with methane emissions from ruminant livestock. Journal of animal science and biotechnology, 8(1), 7. Doi: 10.1186/s40104-017-0141-0

THEODOROU MK, Williams BA, Dhanoa MS, McAllan AB, France J. 1994. A simple gas production method using a pressure transducer to determine the fermentation kinetics of ruminant feeds. Animal feed science and technology. 48: 185-197. Doi: 10.1016/03778401(94)90171-6

WALLACE RJ. 2004. Antimicrobial properties of plant secondary metabolites. Proceedings of the Nutrition Society. 63: 621-629. Doi: 10.1079/PNS2004393

WANG CJ, Wang SP, Zhou H. 2009. Influences of flavomycin, ropadiar, and saponin on nutrient digestibility, rumen fermentation, and methane emission from sheep. Animal Feed Science and Technology. 148(2-4): 157-166. Doi: 10.1016/j.anifeedsci.2008.03.008

ZHANG J, Shi H, Wang Y, Li S, Cao Z, Ji S, He Y, Zhang H. 2017. Effect of dietary forage to concentrate ratios on dynamic profile changes and interactions of ruminal microbiota and metabolites in Holstein heifers. Frontiers in microbiology, 8, 2206. Doi: 10.3389/fmicb.2017.02206 